\title{
From Fox's microspheres into primitive life: An inferencing hypothesis on the origin of life
}

\author{
H. Zhu \\ Center for Integrative Conservation, Xishuangbanna Tropical Botanical Garden, Chinese Academy \\ of Sciences, Mengla, Yunnan 666303, P. R. China \\ *Corresponding author: H. Zhu, e-mail zhuh@xtbg.ac.cn; Fax no.: 86-871-5160916 \\ Short title: Hypothesis on the origin of life
}

\begin{abstract}
The microspheres constituted by proteinoids synthesized from Fox's simulation experiments. They had peptide bond structure and weak catalysis, as well as proliferated themselves. Such microspheres were believed the models for primitive life. Due to lack of metabolism and self-reproduction, the microspheres could not meet requirements of life. Thus, how microspheres could evolved into primitive life remain unsolved mysteries. The microspheres were supposed a dissipative structure and the processes of absorption and hydrolysis could be balanced to maintain their stability by consuming proteinoid. Proteinoid molecules differed in their life spans, which were mainly determined by their multi-space structures. Consequently, molecule selection and retention could occur spontaneously in microspheres and lead to a more organized and stabilized structure of the whole microsphere with time through dissipative process. More complex chain network of chemical reactions could happen in microspheres because the proteinoid with complex, ordered multi-space structure and relatively high catalytic activity would retain. In such microspheres, nucleotides could produce and further aggregate into RNA. The synthesis of real proteins could take place with RNA as the template catalyzed by proteinoids or RNA inside microspheres. When template-based protein molecules replaced the proteinoid inside the microspheres, a protein-based self-catalyzed network of chemical reactions could take place. It is plausible if Fox's proteinoids microspheres is to dawn on a dissipative structure, then molecule selection could occur spontaneously by "dissipative" proteinoids, and the microspheres would
\end{abstract}


acquire catalytic activity due to preserved the proteinoid with a large molecular weight and relatively complex and ordered multi-space structure, and relatively high catalytic activity. Thus the microspheres would spontaneously go to self-organizing, and evolve into primitive life.

Key words: proteinoids; microspheres; dissipative structure; molecule selection; origin of life.

\section{Background}

The question about the origin of life has always been a core issue of philosophy and a long-standing debate between science and religion as well as between materialism and idealism.

From the perspective of materialism, life is a special form of material motion, and an inevitable product of the development and evolution of Earth at a certain stage. Though the origin of life could be achieved through chemical pathways, it was not until the 1950s that the proposition was confirmed when the American scholar Stanley L. Miller [1] successfully synthesized amino acids by simulating primitive Earth conditions.

Later, Sidney W. Fox [2], another American scholar, simulated primitive Earth conditions. When a mixture of various amino acids was heated, polymerization occurred among amino acids and a protein-like hyperpolymer with a molecular weight as high as approximately 8,000 to 20,000 Dalton was produced. Fox called this product a "proteinoid," and further speculated that heat polymerization of amino acids could occur in some "hot zones" on primitive Earth. When these proteinoids were dissolved in water, they would automatically aggregate into a microspheric multi-molecular system, which he termed a microsphere. Microspheres resemble bacteria, have a double-layer boundary, and internal structure. They proliferated themselves through budding and division, and had weak catalysis. Fox believed that such microspheres were the models for primitive bacteria. 
Due to the success of simulation experiments by Miller, Fox, and other researchers, a hypothesis on the origin of life was tentatively unveiled. As investigations went deeper, however, new problems emerged. Though proteinoids synthesized during the simulation experiments had peptide bond structure and weak catalytic action, and Fox even regarded them as protoenzymes, a significant difference was observed when compared with proteinase. Since proteinoids were not synthesized based on a template, the multi-space structure of the molecule had relatively low complexity, poor order, and irregular primary structure. For instance, acidic and basic amino acids in the backbone structure usually formed branched chains at residues. At some positions of the secondary structure, normal $\alpha$-helices could be formed, while at other locations, they could not be formed. Thus, in the secondary and tertiary structures, proteinoids could not develop highly ordered and accurate multi-space structures like proteinase can, and the proteinoid molecules, therefore, failed to obtain high catalytic activity or transitivity. In general, such molecules had weak catalytic activity, and the microspheres constituted by such proteinoids, consequently, also had weak catalytic activity. As we know, metabolism and self-reproduction are two main characteristics of life. Metabolism occurs on the basis of an extremely complex and accurate network of chemical reactions, which require highly accurate and high-speed chemical reactions. Such protein-like molecules, however, failed to meet such requirements. At the same time, biological self-reproduction is jointly achieved with nucleic acids and proteins. In the simulation experiment, it is difficult to synthesize nucleic acids. So far, only oligonucleotides with a small molecular weight can be synthesized in the laboratory. Thus, how microspheres synthesized by the limited catalytic activity of proteinoids evolved into primitive life and achieved self-reproduction, and how nucleic acids and proteins established relationships with each other still remain unsolved 
mysteries.

Proposals for the origin of life remain as hypotheses, meaning they are working assumptions for scientists researching how life began. However, Fox et al. [3] suggested that proteinoid microspheres of appropriate sorts promote the conversion of ATP to adenine dinucleotide and adenine trinucleotide. Other microparticles composed of basic proteinoid and enzymically synthesized poly A cause the conversion of ATP and phenylalanine to various peptides of phenylalanine. When viewed in the context with the origin and properties of proteinoid microspheres, these results model the origin from a protocell of a more contemporary type of cell able to synthesize its own polyamino acids and polynucleotides. Obviously, the hypothesis from Fox et al. is of the most significance to the origin of life [3]. In this article, I make a further inference about how the microspheres could possibly evolve into primitive life.

\section{Inferencing hypothesis of microspheres into primitive life}

I put forward the following hypothesis to investigate how microspheres constituted by proteinoids with the limited catalytic activity synthesized from Fox's simulation experiments [2, 3] evolved into primitive life and achieved self-reproduction, and how nucleic acids and proteins established relationships with each other.

I speculate that microspheres need to go through several processes before evolving into primitive life. First, microspheres acquired relatively high catalytic activity and became activated microspheres, which made it possible for complex networks of reactions to occur inside the microspheres. Then, activated microspheres provided a special micro-environment, which facilitated the occurrence of many complex reactions inside the microspheres that would have 
been difficult in primitive oceans such as the synthesis of nucleic acids. When a certain amount of nucleic acids accumulated inside activated microspheres, proteinoid -catalyzed synthesis of proteins started to occur based on nucleic acid template, then the relationship between nucleic acids and proteins was also established. As template-based proteins gradually replaced proteinoid molecules and the self-catalyzed system for the synthesis of proteins, a nucleic acid template was used, and a qualitative change occurred in activated microspheres. The basic metabolic network of reactions was formed and primitive life emerged.

Given the assumption that under suitable conditions, proteinoid microspheres could have absorbed proteinoid molecules to offset the inevitable natural hydrolysis of proteinoid molecules inside themselves. By consuming proteinoid molecules in the solution, microspheres could grow and maintain the stability of their structure. Thus, as long as there was a sufficient supply of proteinoid molecules in the solution, processes could continue within the microsphere for a long time.

According to the dissipative structure theory proposed by Prigogine \& Nicolis [4], I supposed that such characteristics of microspheres were just features of a dissipative structure. Microspheres are not in a state of equilibrium. Instead, they continuously absorbed proteinoids from the surrounding solution. Since the microsphere could be maintained for a long time, the processes of absorption and hydrolysis could be balanced to maintain the stability of the microsphere structure. Gradually, the microsphere structure could change from disorder to order, evolving towards complication and organization.

How did microspheres evolve into primitive life? On primitive Earth, there were many pathways and approaches that produced proteinoids. Additionally, the proteinoid molecules 
differed in their life spans, which were mainly determined by their multi-space structures. In general, if the molecules were large and their multi-space structure was complex, accurate, and ordered, they would have had a strong capacity of self-protection, leading to resistance to hydrolysis and a long life span. On primitive Earth, the non-template synthesized proteinoid molecules usually had low complexity and order in their multi-space structure. The primary structure of some molecules, however, had relatively long, normal $\alpha$-helices, which led to a relatively complex and ordered multi-space structure, resistance to hydrolysis, and a long life span. Consequently, molecule selection and retention occurred spontaneously in primitive oceans, which was based on the relative difference between the complexity and order of the multi-space structure of proteinoid molecules. Proteinoid molecules with a large molecular weight and relatively complex and ordered multi-space structure would be preserved and accumulated in the primitive oceans due to their resistance to hydrolysis and long life span. Proteinoid molecules without such features would be hydrolyzed. Thus, such preserved and accumulated proteinoid molecules would evolve continuously in the primitive oceans.

Molecule selection inside microspheres was the most significant factor in the origin of life. During the dissipative process in microspheres, proteinoid molecules with a large molecular weight and complex and ordered multi-space structure were absorbed by microspheres, and such molecules would be preserved and accumulated in microspheres due to their stability and long life span. Unstable molecules with a short life span would be decomposed and consumed. Through such processes, simple and unstable molecules would be dissipated, and complex and stable ones would be preserved. Thus, the proteinoid molecules that constituted microspheres became more and more complex and more and more ordered, leading to a more ordered, organized, and 
stabilized structure of the whole microsphere.

It is possible that the catalytic function of large molecules was related to the state of other multi-space structures. The acquisition of catalytic function was determined by a certain highly ordered multi-space structure. Proteins have enzymatic activity due to their folded, highly accurate, and ordered multi-space structure, of which certain locations can form interactions with substrates. This is the same for proteinoid molecules in that proteinoid molecules with a large molecular weight and complex and ordered multi-space structure would have relatively high catalytic activity. Thus, the structure of the microspheres became increasingly complex and ordered during the dissipative process, and microspheres would acquire catalytic activity due to the molecules with high catalytic activity that constituted them.

When the microsphere originally forms, it should be simple and lack catalytic activity, or perhaps be in a near-equilibrium state. Through dissipation of proteinoid molecules, however, it becomes more and more complex and ordered, and its catalytic activity increasingly becomes enhanced. As it moves away from equilibrium, it eventually becomes an activated microsphere. The dissipative structural features of the microsphere, the length of the life span, and the strength of catalytic activity determined by the state of the multi-space structure of proteinoid molecules were compatible and consistent; therefore, it is an inevitable process for the microsphere to evolve into an activated microsphere.

I supposed that inside the activated microsphere, the speed of chemical reactions increased, the direction and the order of chemical reactions were enhanced, and a more complex chain network of chemical reactions even took place. Thus, the activated microsphere created a special micro-environment where many complex chemical reactions could take place that might have 
been unlikely to occur naturally in primitive oceans.

For different activated microspheres, their compositions, structures, degrees of organization, and activation could differ greatly, and the forms and content of chemical reactions could also differ from one microsphere to another, thus creating numerous microenvironments for various chemical reactions to take place. In certain activated microspheres, nucleotides were produced and energy chain reactions took place, which was coupled with the decomposition of organic matter. Nucleotides further aggregated into RNA, short molecules of which could combine with amino acids to produce aminoacyl-RNA. It is possible that inside certain activated microspheres, synthesis of real proteins took place with RNA as the template, aminoacyl-RNA as the amino donor, and proteinoids as the catalyst (perhaps RNA was also involved in catalysis). Thus, the relationship between nucleic acids and proteins might be established before the emergence of primitive life. Template-based proteins had regular peptide bonds, normal $\alpha$-helix secondary structure, and could be folded into a highly ordered multi-space structure, thus had high catalytic activity and specificity. Newly synthesized proteins were continuously integrated into the network of chemical reactions, supplemented and enriched chemical reactions inside microspheres, and gradually replaced proteinoid molecules. When template-based protein molecules replaced all the proteinoid molecules inside the activated microspheres, a highly efficient, protein-based self-catalyzed network of chemical reactions took place, the basic contents of metabolism formed, activated microspheres experienced a qualitative change, and new life eventually emerged. The suggested hypothesis on the origin of life is shown in Figure 1. 


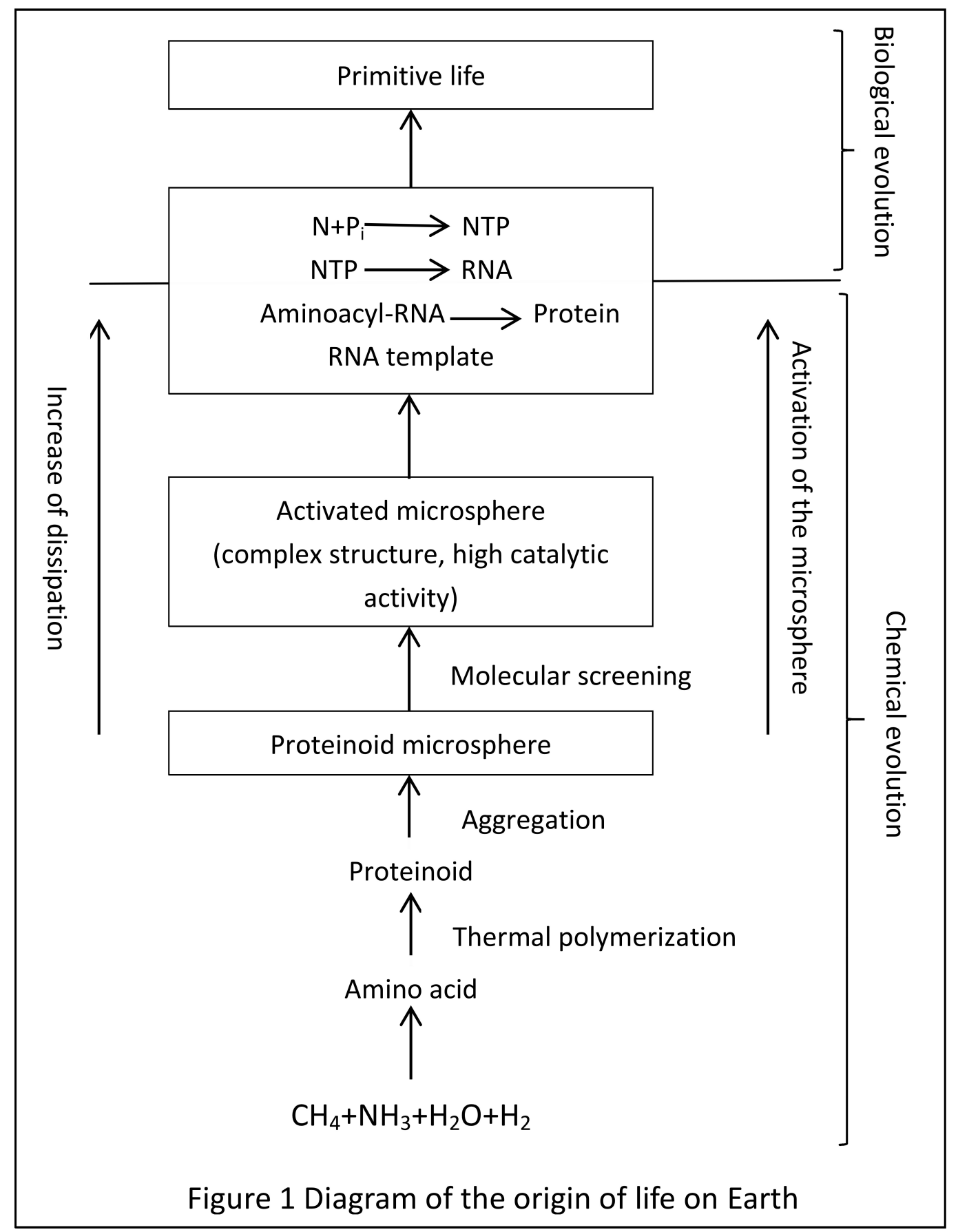

\section{Discussion}

The main idea of my inferencing hypothesis was originally published in Chinese [5], and was similar to several scientists' hypotheses in some aspects and considerations. For example, Huber and Wächtershäuser [6], proposed the possible formation of peptides by activation of amino acids 
in simulation experiments. Huber et al. [7] further put forth a possible primordial peptide cycle. In my hypothesis, due to the dissipative process and molecule selection inside microspheres, more complex chained networks of chemical reactions, including the formation of peptides, could have even took place in microspheres.

Two fundamentally different ideas in the origin of life are: the genetics or replication first scenario $[8,9]$, and the metabolism first scenario [10]. L. E. Orgel [9] stated that a plausible scenario for the origin of life must, therefore, await the discovery of a genetic polymer simpler than RNA and an efficient, potentially prebiotic, synthetic route to the component monomers. His suggestion that relatively pure, complex organic molecules might be made available in large amounts via a self-organizing, autocatalytic cycle might, in principle, help to explain the origin of the component monomers. In my inferencing hypothesis, in certain activated microspheres, nucleotides could be produced, and nucleotides could be further aggregated into RNA, because inside the activated microsphere, the speed of chemical reactions increased, and a more complex chained network of chemical reactions even took place.

RNA molecules had the dual role of catalysts and information storage systems, which provides support for objections to the genetics first scenario [11]. Existing replicators can serve as templates for the synthesis of additional copies of themselves, but they cannot be used for the preparation of the very first such molecule, which must arise spontaneously from an unorganized mixture [12]. Thus, greater attention should be given to metabolism-first theories, which avoid this conflict [12]. F. A. Anet also stated that the reactions were either spontaneous or were catalyzed by inorganic molecules, or by oligo-peptides or proteinoids formed either in a random manner or by mutual catalysis [10]. C. De Duve clarified that protometabolism must have been dependent on 
a network of fairly complex chemical processes and suggested that it was likely that ATP and the other NTPs served as precursors for the synthesis of RNA before participating in the formation of the first RNA molecules [13]. C. De Duve also stated that another property of protometabolism is that it must have relied on a set of robust reactions capable of being maintained over the time needed for enzyme-catalyzed metabolism to arise, which could be as long as several millennia [13]. In my inference, the activated microsphere created a special micro-environment where many complex chemical reactions could take place. It is possible that the synthesis of real proteins could occur with RNA as the template catalyzed by proteinoids or RNA inside certain activated microspheres. My inference is compatible with both Orgel's [8, 9] and Shapiro's [12] ideas. The microspheres were just a dissipative structure and could maintain the stability of their structure to create a special micro-environment where many complex chemical reactions could take place. It is possible that the synthesis of real proteins took place with RNA as the template catalyzed by proteinoids or RNA inside certain activated microspheres. This idea is in the line with De Duve's idea as well.

Carter Jr. \& Wolfenden [14] suggested that genetic coding of 3D protein structures evolved in distinct stages, based initially on the size of the amino acid and later on its compatibility with globular folding in water through their experiments. Wolfenden et al. [15] also suggested that the possible situation could be peptide bond catalyzing formation of RNA, and the interaction between amino acids and nucleotides could be existent before origin of life. These new findings support my idea to some extent.

Baum \& Vetsigian [16] stated that the core of the origin of life problem is to explain the emergence of chemical systems that exhibit the capacity for heritable change and open-ended 
evolution. Once such systems arose, adaptive evolution could take over. They suggested that conducting prebiotic selection experiments should be a priority for the origin of life and hope that scientists from diverse disciplinary backgrounds will design concrete experiments to search life-like chemical systems that show evidence of self-propagation and adaptive evolution. My hypothesis is considerable along these lines.

\section{Conclusion}

The simulation experiments conducted by Fox [2,3] and Nakashima \& Fox [17] produced microspheres that formed automatically by aggregated proteinoids and these proteinoid microspheres promoted the conversion of ATP to adenine dinucleotide and adenine trinucleotide. However, how microspheres synthesized by the limited catalytic activity of proteinoids evolved into primitive life and achieved self-reproduction remain unsolved. Based on the dissipative structure theory proposed by I. Prigogine [4], I supposed that the microspheres were features of a dissipative structure. Molecule selection could occur spontaneously in microspheres. Proteinoid molecules with a large molecular weight and relatively complex and ordered multi-space structure would be preserved and accumulated due to their resistance to hydrolysis and relatively long life span. Thus, the microspheres would acquire catalytic activity due to the molecules with relatively high catalytic activity that were retained through the dissipative process, eventually becoming “activated microspheres." The activated microsphere created a special micro-environment. Inside the activated microsphere, the speed of chemical reactions increased, and a more complex chain network of chemical reactions even took place. In certain activated microspheres, nucleotides could be produced, and nucleotides could further aggregate into RNA. It is possible that the synthesis of real proteins took place with RNA as the template and proteinoids as the catalyst 
(perhaps RNA was also involved in catalysis) inside certain activated microspheres. Thus, the relationship between nucleic acids and proteins might be established before the emergence of primitive life.

\section{Conflict of interest statement}

Nothing declared.

\section{Acknowledgments}

This project was funded by The National Natural Science Foundation of China (41471051, 31170195, 41071040). English editing was completed by TopEdit (www.topedit.cn).

\section{References}

1 Miller SL. Production of amino acids under possible primitive earth conditions. Science 1953, 117 (3046): 528-529.

2 Fox SW. The origins of prebiological systems and of their molecular matrices. New York: Acad. Pr. 1965.

3 Fox SW, Jungck JR, Nakashima T. From proteinoid microsphere to contemporary cell: formation of internucleotide and peptide bonds by proteinoid particles. Origins of Life 1974, 5(1): 227-237.

4 Prigogine I, Nicolis G. Self-Organization in Non-Equilibrium Systems. Wiley. ISBN 0-471-02401-5. 1977.

$5 \mathrm{Zhu}$ H. A hypothesis on the origin of life. Science (KEXUE) 1991, 43(1): 52-52, 11 (in Chinese). 
6 Huber C, Wächtershäuser G. Peptides by activation of Amino Acids with CO on (Ni,Fe) S Surfaces: Implications for the Origin of Life. Science 1998, 281: 670-672.

7 Huber C, Eisenreich W, Hecht S, Wächtershäuser G. A possible primordial peptide cycle. Science 2003, 301(5635): 938-940.

8 Orgel LE. Prebiotic chemistry and the origin of the RNA world. Crit Rev Biochem Mol Biol 2004, 39: 99-123.

9 Orgel LE. Self-organizing biochemic alcycles. PNAS 2000, 97(23): 12507.

10 Anet FA. The place of metabolism in the origin of life. Curr Opin Chem Bio 2004, 18: 654-659.

11 Gilbert W. Origin of life: The RNA world. Nature 1986, 319: 618.

12 Shapiro R. A replicator was not involved in the origin of life. IUBMB Life 2000, 49:

$173-176$.

13 De Duve C. A research proposal on the origin of life. Origins Life Evol Biosphere 2003, 33 : $559-574$.

14 Carter CW, Wolfenden R. tRNA acceptor stem and anticodon bases form independent codes related to protein folding. PNAS 2015, 112(24): 7489-7494.

15 Wolfenden R, Lewis CA, Yuan Y, Carter CW. Temperature dependence of amino acid hydrophobicities. PNAS 2015, 112 (24), 7484-7488.

16 Baum D, Vetsigian K. An experimental framework for generating evolvabel chemical systems in the laboratory. Orig Life Evol. Biosph 2017, 47: 481-497.

17 Nakashima T, Fox SW. Formation of peptides from amino acids by single or multiple additions of ATP to suspensions of nucleoproteinoid microparticles. Biosystems 1981, 14(2): $151-161$. 